\title{
A computer virus spreading model with nonlinear infectivity on scale- free network
}

\author{
Changsong Deng ${ }^{1}$, Qiming Liu $^{2}$ \\ ${ }^{1}$ Section of Fundamentals, Ordnance Engineering College, Shijiazhuang 050003, China
}

Keywords. Scale-Free network, computer viruses, nonlinear infectivity, threshold

\begin{abstract}
In this paper, a novel epidemic model of computer virus on scale-free network with nonlinear infectivity is proposed. The spreading dynamics of the virus was analyzed. The spreading critical threshold for the model was presented. Theoretical analyses indicate that the outbreak of the virus is entirely determined by the threshold. Numerical simulations confirmed the analytical results.
\end{abstract}

\section{Introduction}

Internet provides conveniently various services and information to people. Meanwhile, the easy access and wide usage of Internet make it a primary target for viruses. In the past, outbreaks of computer viruses have brought about huge financial losses. As the major means of defending against viruses, antivirus software is more a technique than a science; it cannot predict the evolution of viruses and hence, cannot provide global prevention/control policies. With the dramatic increase in the number of virus, relying solely on measures to clear the viruses can not meet the ultimate needs. Facing the severe situation of virus' destroy, it's urgent for people to establish epidemic model, explore virus propagation characteristics and advance controlling schemes in the network information security field.

The computer viruses bear a striking resemblance to their biological counterparts. Cohen [1] and Murray [2] inventively suggested to exploit the tools developed in epidemic dynamics of infectious diseases to study the spreading behavior of computer viruses. Following this idea, Kephart and White [3] established the first epidemic model of computer viruses. From then on, most epidemic models usually have been proposed based on the assumption that Internet is homogeneous network [4-6]. At the end of last century empirical studies report that Internet has scale-free properties [7-9]. Studies on epidemic spreading in SF networks present us with surprising result, such as absence of any epidemic threshold, hierarchical spread of epidemic outbreaks [10-12], and so on. Most previous work toward this direction was limited to three simplistic models: the SI model [13-14], the SIS model [15-16] and the SIR model [17-18].

However, most previous works mainly focus on the impact of the underlying topology and assume that the transmission is uniformly distributed among all links, that is, each infected individual will try to contact all its susceptible neighbors once within one time step. In fact, this kind of uniform transmission is induced from the assumption that each node's potential infectivity, counted by its possible maximal contribution to the propagation process within one time step, is strictly equal to its degree, i.e., 'infectivity =degree'. The node with very large degree is called hub in computer networks, whereas the node with great infectivity in epidemic contact networks is termed super-spreader. Thus, the above assumption means hub =super-spreader. This assumption fails to consider that the infectivity of an infected computer is limited, because computer's processing capacity and network bandwidth are limited.

In this paper, we aim to overlook the above defects and understand the spreading behaviors of computer viruses in Internet. We present a novel SIDS (susceptible-infected-detached-susceptible)

epidemic model of computer virus to investigate the impact of nonlinear infectivity in Internet with the scale-free property. We obtain the corresponding spreading threshold and analyze the globally dynamic behaviors of the virus. Then, the numerical simulations are given, which are well consistent with the theoretical results.

The remainder of this paper is structured as follows. In Section 2, we will briefly formulate this novel epidemic model. Then in Section 3, we determine the threshold value and analyze the 
globally dynamic behaviors of the virus. In Section 4, we carry out extensive numerical simulations to verify the theoretical analysis in Section 3. At last, in Section 5 we summarize this work.

\section{Model formulation}

It is well known [11-13] that the node degrees of Internet asymptotically follow a power law distribution, $P(k) \square k^{-\gamma}$, where $P(k)$ stands for the probability that a node chosen randomly from Internet is of degree $k$. For this model in Internet, each computer is represented by a vertex of the network and the edges are communication links between them, along which the infection may spread. Let $S_{k}(t), I_{k}(t)$ and $D_{k}(t)$ be the densities of susceptible, infected, and quarantined vertexes of degree $k$ at time $t$. Obviously, they must satisfy the normalized condition $S_{k}(t)+I_{k}(t)+D_{k}(t)=1$ if the total number of the node is fixed. The infection transmission is defined by the spreading rate $\lambda$, at which each susceptible computer acquires the infection from an infected neighbor during one time step. An infected computer goes through a quarantine period firstly before becoming susceptible again. The rate constant of quarantine for infected computers is denoted by $\mu$. $\tau$ is the average quarantine period. All parameters are positive. Then by applying the mean-field technique to the above assumptions, we have the following dynamics model based on delay differential equations

$$
\left\{\begin{array}{l}
\frac{d S_{k}(t)}{d t}=-\lambda k S_{k}(t) \Theta(t)+\sigma D_{k}(t) \\
\frac{d I_{k}(t)}{d t}=-\mu I_{k}(t)+\lambda k S_{k}(t) \Theta(t) \\
\frac{d D_{k}(t)}{d t}=\mu I_{k}(t)-\sigma D_{k}(t)
\end{array}\right.
$$

$\Theta(t)$ denotes the probability from any given link to an infected node. It satisfies the relation

$$
\Theta(t)=\frac{\sum_{k=1}^{n} \varphi(k) P(k) I_{k}(t)}{\langle k\rangle}
$$

$\varphi(k)$ denotes the nonlinear infectivity related with the degree of an infected node, where $\varphi(k)=\alpha k^{\alpha} / 1+b k^{\alpha}, 0 \leq \alpha \leq 1, a>0, b \geq 0[15] .\langle k\rangle$ is the average degree within the network.

\section{The analysis of the model}

Let

$$
\lambda_{c}=\frac{\langle k\rangle}{\langle k \varphi(k)\rangle}
$$

Theorem 1. There is always a virus-free equilibrium $E_{0}\{(1,0,0)\}_{k}$ for system (1) and when $\lambda / \mu>\lambda_{c}$ system (1) has a unique endemic equilibrium $E_{*}\left\{\left(S_{k}^{*}, I_{k}^{*}, D_{k}^{*}\right)\right\}, S_{k}^{*}, I_{k}^{*}, D_{k}^{*}$ denote the densities of susceptible, infected, and quarantined vertexes of degree $k(k=1,2, \cdots n)$.

Proof. It is easily verified that $E_{0}$ is always an equilibrium of system (1). To get the equilibrium solution ${ }^{E_{*}}$ we need to make the right side of system (1) equal to zero. Then the equilibrium ${ }^{E_{*}}$ should satisfy 


$$
\left\{\begin{array}{l}
-\lambda k S_{k}^{*} \Theta^{*}+\sigma D_{k}^{*}=0 \\
-\mu I_{k}^{*}+\lambda k S_{k}^{*} \Theta^{*}=0 \\
S_{k}^{*}+I_{k}^{*}+D_{k}^{*}=1
\end{array}\right.
$$

where

$$
\Theta^{*}=\frac{1}{\langle k\rangle} \sum_{k} \varphi(k) P(k) I_{k}^{*}
$$

Solving Eq.(4) yields

$$
I_{k}^{*}=\frac{\sigma}{\sigma+\lambda k \Theta^{*}}
$$

Substituting Eq.(6) into Eq.(5), we can obtain the self-consistent equation about $\Theta^{*}$ as follows

$$
\Theta^{*}=\frac{\sum_{k=1}^{n} \varphi(k) P(k) I_{k}^{*}}{\langle k\rangle}=\frac{1}{\langle k\rangle} \sum_{k=1}^{n} k \varphi(k) P(k) \frac{\sigma \lambda \Theta^{*}}{\sigma \mu+\lambda k \Theta^{*}}=f\left(\Theta^{*}\right)
$$

Apparently, $\Theta^{*}=0$ is always a trivial solution, which denotes the virus-free state. Therefore, a nontrivial solution exists provided

$$
f\left(\Theta^{*}\right)=\frac{1}{\langle k\rangle} \sum_{k=1}^{n} k \varphi(k) P(k) \frac{\sigma \lambda \Theta^{*}}{\sigma \mu+\lambda k \Theta^{*}}=0
$$

Since

$$
\frac{d f\left(\Theta^{*}\right)}{d \Theta^{*}}>0, f(1)>0
$$

a nontrivial solution exists if and only if $f(0)<0$, i.e., $\lambda / \mu>\lambda_{c}$.

Substituting the nontrivial solution of $f(\Theta)=0$ into Eq.(6), we can get $I_{k}^{*}$. By Eq.(4) and (6), we can easily obtain that

$$
0<S_{k}^{*}<1,0<I_{k}^{*}<1,0<D_{k}^{*}<1
$$

Therefore, the equilibrium $E^{*}$ is well-defined. Hence, when $\lambda / \mu>\lambda_{c}$, one and only one endemic equilibrium of system (1) exists. This completes the proof.

IV. Numerical simulation

Extensive numerical simulations are carried out on BA scale-free model to demonstrate the above mentioned theorems. The degree distribution is $P(k)=c k^{-\gamma}$, and constant ${ }^{c}$ satisfies $\sum_{k=1}^{n} P(k)=1$. Here we set the maximum degree $n=100$, the minimum degree $m=1$.

Consider system (1) with parameters $\lambda=0.2, \mu=0.8, \sigma=0.1, a=6$,

$b=1, \alpha=0.6$, which runs on a scale-free network with $\gamma=2.5$. Then $\lambda_{c}=0.253>\lambda / \mu$. For a set of initial conditions satisfying $I_{k}(0)=0.001, S_{k}(0)=0.999, D_{k}(0)=0$, Fig. 1 shows the evolutions of $I_{10}(t), I_{55}(t), I_{85}(t)$, from which it can be seen that viruses will tend to extinction, in agreement with Theorem 1. 


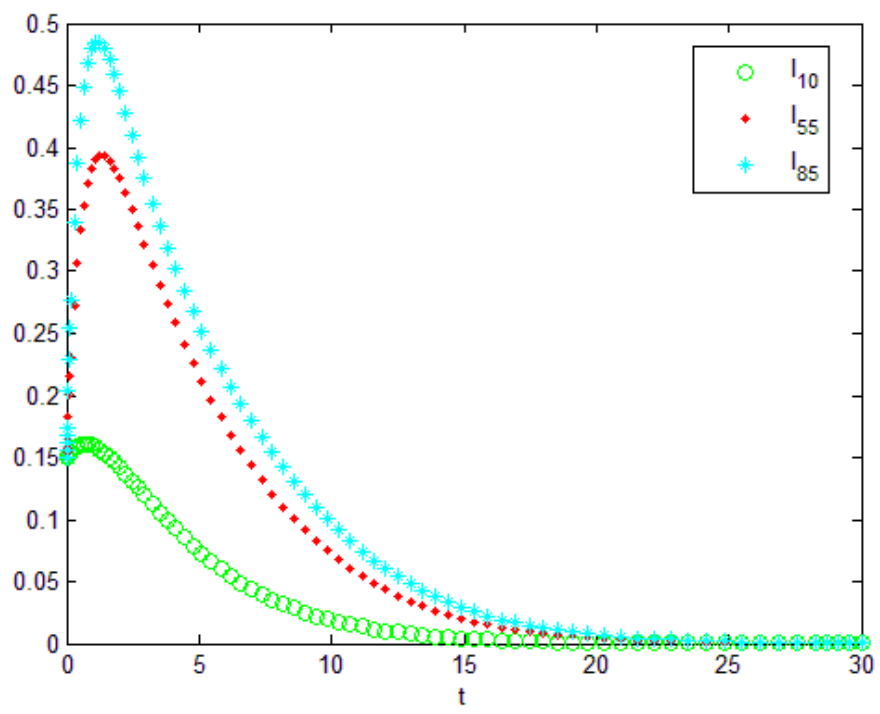

Figure 1: Evolutions of $I_{10}(t), I_{55}(t), I_{85}(t)$, for the system (1) with condition $\lambda / \mu<\lambda_{c}$.

Consider system (1) with parameters $\lambda=0.4, \mu=0.8, \sigma=0.1, a=6$,

$b=1, \alpha=0.6$, which runs on a scale-free network with $\gamma=2.5$. Then $\lambda_{c}=0.253<\lambda / \mu$. For a set of initial conditions satisfying $I_{k}(0)=0.001, S_{k}(0)=0.999, D_{k}(0)=0$, Fig. 2 shows the evolutions of $I_{10}(t), I_{55}(t), I_{85}(t)$, from which it can be seen that viruses will tend to extinction, in agreement with Theorem 1.

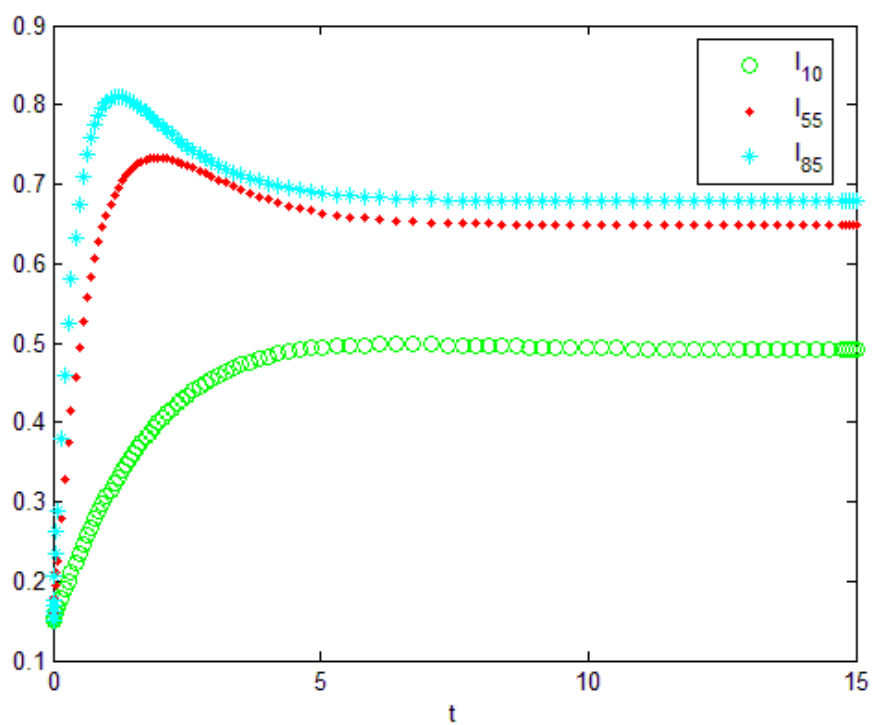

Figure 2: Evolutions of $I_{10}(t), I_{55}(t), I_{85}(t)$, for the system (1) with condition $\lambda / \mu>\lambda_{c}$.

\section{Conclusion}

In summary, to better understand the effect of the time delay on the spread of computer viruses, we introduce a novel SIQS model capturing the epidemics of computer viruses on scale-free networks which includes the nonlinear infectivity and quarantine delay. The spreading threshold for the model has been determined. The global asymptotic stability of the virus-free equilibrium has been shown when the threshold is below one, whereas the permanence of the virose equilibrium has been proved if the threshold is above one. These results help to work out policies of inhibiting computer viruses. 


\section{References}

[1] F. Cohen, Computer viruses: theory and experiments, Comput. Secur. 1987, 6(1): 22-35.

[2] W. H. Murray, The application of epidemiology to computer viruses, Comput. Secur. 1988, 7(2): 130-150.

[3] J.O. Kephart, S. R. White, Directed-graph epidemiological models of computer viruses, in: Proc. 1991 IEEE Comput. Soc. Symp. Res. Secur. Privacy, 1991: 343-359.

[4] X. Han, Q. Tan, Dynamical behavior of computer virus on Internet, Appl. Math. Comput. 2010, 217(6): 2520-2526.

[5] B. K. Mishra, S. K. Pandey, Dynamic model of worms with vertical transmission in computer network, Appl. Math. Comput. 2011, 217(21): 8438-8446.

[6] J. Ren, X. Yang, L. X. Yang, et al, Adelayed computer virus propagation model and its dynamics, Chaos Soliton Fract. 2012, 45(1): 74-79.

[7] Barab' asi A L, Albert R, et al. Emergence of scaling in random networks. Science, 1999, 286: 509-512.

[8] M. Faloutsos, P. Faloutsos, C. Faloutsos, On power-law relationships of the Internet topology, in: ACM SIGCOMM Comput. Commun. Rev. 1999, 29(4): 251-262.

[9] R. Albert, Barab' asi, A L, Statistical mechanics of complex networks, Rev. Mod. Phys. 2002, 74(1): 47-97.

[10] R. Pastor-Satorras, A. Vespignani, Epidemic spreading in scale-free networks, Phys. Rev. Lett. 2001, 86(14): 3200-3203.

[11] R. Pastor-Satorras, A. Vespignani, Epidemic dynamics and endemic states in complex networks, Phys. Rev. 2001, 63(6): 066117.

[12] M. Barth' elemy, A. Barrat, R. Pastor-Satorras, et al, Velocity and hierarchical spread of epidemic outbreaks in scale-free networks, Phys. Rev. Lett. 2004, 92(7): 178701.

[13] T. Zhou, J. G. Liu, W. J. Bai, et al, Behaviors of susceptible-infected epidemics on scale-free networks with identical infectivity, Phys. Rev. E. 2006, 74(5): 056109.

[14] M. Karsai, M. Kivel “' a, R. K. Pan, et al, Small but slow world: how network topology burstiness slow down spreading, Phys. Rev. E. 2011, 83(2): 025102.

[15] H. Zhang, X. Fu, Spreading of epidemics on scale-free networks with nonlinear infectivity, Nonlinear Analysis. 2009, 70: 3273-3278.

[16] G. H. Zhu, X. C. Fu, G. R. Chen, Global attractivity of anetwork-based epidemic SIS model with nonlinear infectivity, Commun. Nonlinear Sci. Numer. Simul. 2012, 17(6): 2588-2594.

[17] C. Y. Xia, L.Wang, S. W. Sun, et al, An SIR model with infection delay and propagation vector in complex networks, Nonlinear Dyn. 2012, 69: 927-934.

[18] H. L. Zhang, Z. H. Guan, T. Li, A stochastic SIR epidemic on scale-free network with community structure, Physica A. 2013, 3 